\title{
Intelligent Management System of Electric Power Meter Based on Analysis of the Index
}

\author{
DU Jie ${ }^{1 *}$, CHENG Yingying ${ }^{1}$, XIAO $\mathrm{Ji}^{1}$, ZHOU Feng ${ }^{1}$, YANG Huaxiao ${ }^{1}$ \\ ${ }^{1}$ Chongqing Electric Power Research Institute, Chongqing 401123, China
}

Keywords: Electric power meter, analytic hierarchy process, fuzzy comprehensive evaluation, operating condition, test strategy

Abstract. Currently, the operation management of energy meter is still using regular maintenance and maturity rotation mode. With the recent popularity of electronic power meter, new online monitoring methods for electric power meter have improved greatly. In this background evaluation model for electric power meter has been built. This model has not only considered the traditional foundation data and Field test data, but also considered the new online monitoring method for energy meter, putting forward an index system in three aspects that reflect energy meter's condition sufficiently. And we study every index's influence, using FAHP to solve every index weight and evaluate energy meter's condition. Based on the evaluation result, we can make energy meter's test and alternation strategy to adjust their period dynamically. Using electric power communication network, we can realize intelligent management of energy meter easily.

\section{Introduction}

Energy metering devices as a core part of power companies and trade settlement clients plays an important role in energy metering. They ensure reliable and accurate operation of the power grid aspect of great significance. Energy metering devices consists of meter, voltage transformers, current transformers, secondary circuit composed of four parts.

Currently, for the management of meter is still used in regular maintenance and maturity rotation manner ${ }^{[1]}$. With the recent advances in electronic technology, electromagnetic induction meter has gradually been replaced by electric power meter, smart meter. In addition, construction of smart grid promotes the development of remote meter reading, on-line monitoring and other technical means. In this context, the traditional meter test and the cycle of rotation no longer meet the new requirements. Part of the well-run meter expires forced rotation, resulting in a lot of waste of human and material resources, but also increases the chance to generate new risks, reducing the reliability of measurement. Partially because of defective meter not yet reached the periodic inspection time, it failed to detect flaws and shortcomings, leading to errors and result in losses ${ }^{[2]}$.

Therefore, for the shortcomings of the current management and insufficient power meter, proposed and established meaning a new energy management system table status is very important. In this paper, we selected a series index to fully reflect the meter running status indicators, an index system. This information combines basic information, on-site inspection information and new online monitoring tools. By analyzing the impact of the size of the index, and then use the FAHP process to obtain the index weight, build quantitative evaluation model. Finally, we developed a new rotation and inspection strategy; you can achieve a dynamic test and adjust the cycle of rotation meter.

This management system can be easily implemented in a timely manner to adjust each cycle of rotation in the inspection and meter operation, in line with the trend of intelligent power equipment operation and management, with a high promotional value.

\section{Comprehensive Evaluation System}

Basic information and site inspection can reflect the basic information of electric power meter. Online monitoring system can also provide a number of operational statuses to reflect the quality of their data. Choose from three aspects indicators to assess the state of the meter, it can fully reflect its full operational status, assessment results more scientific and reasonable. 


\section{Basic Information}

Basic information covers the status of the merits of index information offline, which is divided into two groups: configuration mode and laboratory accreditation.

\section{Online Monitoring Information}

Operational monitoring indicators covering information meter running through online monitoring tools such as SG186, information collection systems can be observed or recorded to reflect the status of the meter station ${ }^{[3]}$. Online monitoring information contained operation, operating environment, operational monitoring three categories.

\section{Site Testing Information}

Site inspection covering index information when operating personnel on-site verification of the meter measured taken. On-site detection information indicators include: on-site inspection error, error combination, clock checking $(1 \sim 5 \mathrm{~min})$.

\section{Meter Status Assessment Model}

Meter state evaluation system has 42 indicators, while each of the indicators in the evaluation of the extent of the power meter state importance are not the same proportion. Therefore, the study analyzed the size of each indicator of the degree of influence through the scientific method, to establish its share in the corresponding evaluation model weights is very necessary ${ }^{[4]}$.

At present, the commonly used indicators of empowerment methods can mainly be divided into two kinds of objective and subjective. Objective weighting method based on the relationship between the original data is determined by the weight of certain mathematical algorithms; mathematical theory there is a strong basis for its assessment of the results does not depend on subjective judgments. However, the objective weighting method depends on sufficient sample data and the actual problem domain, versatility and participation of the poor, the calculation method is also more complex, and does not reflect the judge's emphasis on the different properties of indicators, sometimes fixed weights will the actual importance of the property were quite different. Since the meter status indicators on the overall assessment of the impact depends on the expertise, so objective weighting method does not apply to this project.

Fuzzy Analytic Hierarchy Process (FAHP) is a subjective weighting method. It incorporates the concept of fuzzy mathematics; we can qualitative and quantitative evaluation of handling a variety of factors. Its characteristics are the subjective judgment process of mathematical thinking of, so that decision making is easy to be accepted. Therefore, FAHP can fit complex situation in the field of social sciences. Since FAHP has completeness, rigor, simplicity, it has been widely used in all walks of life. Specific methods measures are as follows.

\section{The Establishment of a Hierarchy Model U}

Establish a hierarchy model is the basis of fuzzy analytic hierarchy process. Hierarchies' evaluation index system also determines the weights were calculated using fuzzy analytic hierarchy process is more appropriate.

Let one evaluation $G \mathrm{n}$ sub-index covers down, the index configuration set $U=\left\{U_{1}, U_{2}, \ldots\right.$, $\left.\mathrm{U}_{\mathrm{n}}\right\}$ 。

Set $U$ of i-th element Ui contains s sub indicators: $U i=\left\{U_{i 1}, U_{i 2}, \ldots, U_{\text {in }}\right\}$

\section{Construct Indicators Fuzzy Complementary Judgment Matrix R}

Fuzzy AHP requires drill influence the relative importance of interlink ages between the elements of the calculation, and to quantify the composition of fuzzy complementary judgment matrix, as a basis for analysis.

In order to assess the state of the system "Meter Status" for example, as a target G. Analysis This index contains the operating conditions, operating environment, operational monitoring three indicators. Determine the impact of these factors need to "Meter Status" and given appropriate weight. The relative importance of each of the sub-factors of pair wise comparison, the comparison result as a 
constituent element of fuzzy complementary judgment matrix $r_{i j}$, matrix structure called G's impact on the target judgment matrix element, as shown below.

$$
R=\left[\begin{array}{cccc}
r_{11} & r_{12} & \mathrm{~L} & r_{1 n} \\
r_{21} & r_{21} & \mathrm{~L} & r_{2 n} \\
\mathrm{M} & \mathrm{M} & \mathrm{O} & \mathrm{M} \\
r_{n 1} & r_{n 2} & \mathrm{~L} & r_{n n}
\end{array}\right]
$$

$r_{i j}$ represents the relative terms of the relative importance index $\mathrm{G}_{\mathrm{i}} \mathrm{Uj}$ of indicators. Table 1 shows the assignment criteria to determine the matrix elements.

Table1. The criterion of judgment matrix

\begin{tabular}{ccc}
\hline Scaling & Meaning & Remark \\
\hline 0.1 & Index $U_{i}$ is not absolutely important than Index $U_{j}$ & $0.2,0.4,0.6,0.8$ \\
0.3 & Index $U_{i}$ is not obviously important than Index $U_{j}$ & are critical value \\
0.5 & Index $U_{i}$ is equally important with Index $U_{j}$ & \\
\hline 0.7 & Index $U_{i}$ is obviously important than Index $U_{j}$ & \\
\hline & Index $U_{i}$ is absolutely important than Index $U_{j}$ & \\
\hline
\end{tabular}

\section{Consistency Checking and Adjustment}

It gives a certain degree of consistency judgment matrix additive index $\rho$, with a $\rho$ value judgment matrix test whether satisfactory additive consistency, the greater the $\rho$ value, the worse the consistency of judgment matrix R, $\rho$ 's value is given by:

$$
\rho=\frac{2}{n(n-1)(n-2)} \sum_{i=1}^{n-1} \sum_{j=i+1}^{n} \sum_{\substack{k+1 \\ k \neq i, j}}^{n}\left|r_{i j}-\left(r_{i k}+r_{k j}-0.5\right)\right|
$$

If $\rho$ is less than a preset threshold, it is considered to satisfy the judgment matrix consistency requirements, or need to re-adjust them. Usually the threshold value is set at 0.5 .

\section{Calculate the Index Weight}

Let fuzzy complementary judgment matrix $R=\left(r_{i j}\right)_{n \times n}$.

$\mathrm{R}$ matrix rows sum. Let $r_{i}=\sum_{j=1}^{n} r_{i j}, r_{i j}^{*}=\frac{r_{i}-r_{j}}{2(n-1)}+0.5$.

rij* composite the Matrix $\mathrm{R} * \mathrm{R} *$ matrix of rows and normalized to obtain index weight vector $w=\left(w_{1}, w_{2}, \cdots, w_{n}\right)^{T}$.among them

$$
w_{i}=\frac{\sum_{j=1}^{n} r_{i j}+\frac{n}{2}-1}{n(n-1)}
$$

Calculated for each indicator in the weights, it can obtain energy meter state score by weighted sum method, as the results of the assessment.

State of mind Meter score of $\mathrm{S}$, there 


$$
S=\sum_{i=1}^{n} w_{i}^{\prime} x_{i}
$$

Depending on the state score, it can be divided into different states meter intervals to provide a basis for the development of state inspection and rotation strategies.

Table2 .Evaluation interval of electric power meter operation condition

\begin{tabular}{cc}
\hline Meter operation condition & Score range \\
\hline Good & {$[85,100]$} \\
Normal & {$[65,85)$} \\
Note & {$[40,65)$} \\
Abnormal & {$[0,40)$} \\
\hline
\end{tabular}

\section{Meter State Inspection and Rotation Strategies}

Meter based on the state of the evaluation results, we propose a new state energy meter testing and rotation strategies. Give full consideration to the importance of the new strategy different categories of energy meter, combined with the newly established power meter line monitoring tools (such as user information collection system, measurement production scheduling platform), periodic inspection of state also developed a corresponding program. State inspection is an online monitoring system application such as energy metering device information collection system, measurement production scheduling platforms. It can run on the part of energy metering device parameters such as current, voltage, frequency, power factor, and running images, abnormal event records to conduct an inspection and examination and determine whether it is in a reasonable state of operation.

\section{Summary}

This paper analyzes the current energy performance management system deficiencies, in the new situation of the current development of smart grid construction, proposed and established a state energy meter evaluation system. The system not only selected basic information and site inspection meter data, taking into account the growing maturity of on-line monitoring techniques meter can bring a lot of reaction energy meter running state data. Condition Assessment Model to the index system based on the establishment meter using fuzzy analytic hierarchy process.

On the basis of meter on the state assessment results to develop a new energy meter state inspection and rotation strategy, dynamic test and adjust the cycle of rotation meter to solve the existing meter management system, operation and maintenance of low efficiency, excessive rotation problem. The use of internal power system communication network, a number of indicators data fusion, which can achieve the meter intelligent management.

\section{References}

[1] Management system [D]. University of Electronic Science and Technology, 2013.

[2] Peng Ling. Asset management of energy metering device [J] .Chinese high-tech enterprises, 2015,01: 185-187.

[3] Lu Donghai, Sun Chunjun, Wang Xiaohu. Smart Substation on-line monitoring system [J]. Electric Power Automation Equipment, 2011,01: 134-137.

[4] Luo Sijia, Liao Ruijin, Wang Youyuan, Liu Ling. Power Transformer Condition with Variable Weights fuzzy comprehensive evaluation [J]. High Voltage Engineering, 2007, (08): 106-110. 